\title{
IDENTIFICACIÓN DE OPORTUNIDADES PARA LA MEJORA DE LA SEGURIDAD Y SALUD OCUPACIONAL EN LAS PYMES DE REPÚBLICA DOMINICANA (PRIMERA PARTE)
}

Identifying Opportunities for Improvement of Occupational Safety and Health in SMEs Dominican Republic

\section{Yuri Durán*}

Resumen: Este artículo examina los requisitos exigidos por el Ministerio de Trabajo en su Reglamento de Seguridad y Salud en el Trabajo, conocido como Reglamento 522-06, para la presentación de Programas de Seguridad y Salud, comparando dichos requisitos con regulaciones y experiencias de otros países en la mejora de la prevención de riesgos laborales en la pequeña y mediana empresa. Se concluye que los costos de cumplimiento con los requisitos del Programa de Seguridad y Salud, basado en los 20 elementos establecidos por el Reglamento 522-06, hacen difícil su implementación. Recomienda modificaciones del Reglamento 522-06 a los fines de facilitar el cumplimiento de las Pymes con el mismo y la implementación de programas para mejorar la inversión de los fondos previstos por el seguro de riesgos laborales para la promoción de la prevención.

Palabras clave: Seguridad, salud en las PYMES, salud ocupacional, República Dominicana.

Catedrático de Maestría en Prevención de Riesgos Laborales de INTEC. Correo electrónico: yuri.duran@intec.edu.do 
Abstract: This article examines the requirements of the Ministry of Labor in its Regulation for Safety and Health at Work, known as Regulation 522-06, for presentation Programs of Safety and Health, comparing these requirements with regulations and experiences of other countries in improving prevention of occupational hazards in small and medium enterprises. We conclude that the costs of compliance with the requirements of Health and Safety Program based on the 20 elements established by Regulation 522-06 make it difficult to implement. Recommended amendments to Regulation 522-06 in order to facilitate compliance therewith SMEs and implementation of programs to improve the investment of the funds provided by insurance occupational hazard for promoting prevention.

Keywords: Safety, Occupational Health in SMEs, Occupational Health, Dominican Republic.

\section{Introducción}

\subsection{Descripción}

Hasta el 17 de octubre de 2006, fecha en que mediante el decreto 522-06 se pone en vigencia el Reglamento de Seguridady Salud en el Trabajo, la implementación de programas de prevención de riesgos laborales en las empresas de República Dominicana careció de una legislación actualizada que impulsó al Ministerio de Trabajo a exigir sistemas de gestión de la seguridad y salud en las mismas. Esta investigación, llevada a cabo entre abril de 2014 y marzo de 2015, procuró evaluar el impacto de este Reglamento en la mejora de las condiciones de trabajo en las empresas dominicanas. 
Identificación de oportunidades para la mejora de la seguridad y salud ocupacional en las Pymes de República Dominicana (primera parte)

\subsection{Justificación}

El Reglamento de Seguridady Salud en el Trabajo (522-06), al establecer los 20 elementos que deberían cumplir los programas de seguridad y salud, no definió contenidos específicos para los mismos. Es en el año 2012 cuando la Dirección General de Higiene y Seguridad Industrial del Ministerio de Trabajo emitió los términos de referencia para su preparación; sin embargo, en ninguno de los casos se delimitaron requisitos según los tamaños de empresa o sus capacidades económicas.

Es de ahí que nace la necesidad de producir un documento complementario donde, sin disminuir las garantías que requiere el trabajador de un espacio con los riesgos laborales controlados, el programa esté en un plano realizable y no ponga en riesgo la recertificación de las empresas que bajo el amparo de lo exigido por el artículo 8 del Reglamento 522-06 se hayan certificado, bajo la promesa de cerrar brechas a su cumplimiento.

Además de lo anterior, la determinación de los costos de aplicación del Reglamento 522-06 es una información importante para los órganos de decisión que tienen el potencial de financiar programas pilotos en sectores con limitaciones económicas y con altos riesgos laborales.

\subsection{Hipótesis}

El cumplimiento con los mandatos del reglamento 522-06 se va reduciendo a medida que disminuye la capacidad económica de las empresas, lo cual se convierte en una especie de dumping social en el que salen perjudicados los trabajadores de las mismas, además de que es motivo de quejas para las que están en cumplimiento. 


\subsection{Tesis}

La República Dominicana requiere de instrumentos que faciliten el cumplimiento, por parte de las Pymes, de los requisitos del Reglamento de Seguridad y Salud en el Trabajo (522-06) de una forma costo - efectiva.

\subsection{Objetivos}

Los objetivos de esta investigación fueron:

- Desarrollar un modelo para la formulación de Programas de Seguridad y Salud en las Pymes, con base a requerimientos del Reglamento de Seguridady Salud en el Trabajo (522-06).

- Establecer el costo de cumplir con el Reglamento de Seguridad y Salud en el Trabajo (522-06) para las Pymes que operan en República Dominicana.

- Identificar oportunidades de reducción de los costos sociales y económicos de la accidentalidad para la República Dominicana.

\subsection{Metodología}

La investigación fue desarrollada a partir del análisis de las estadísticas nacionales de trabajo y producción, a fin de determinar la representatividad que tienen las Pymes en los diversos sectores donde inciden y en los cuales la Administradora de Riesgos Laborales (ARLSS) clasifica sus datos. Con estos se identificaron 20 Pymes representativas de su actividad económica y tamaño del Universo. Esta selección se hizo de forma empírica, con muestreo intencional u opinático, con muestras racionadas o intencionadas.

Mediante la combinación de la observación sistemática y estructurada, participante, en equipo, con trabajo de campo, y la revisión de documentos escritos (fuentes históricas, informes, estudios, memorias, anuarios y documentos oficiales) y estadísticos oficiales, 
de fuentes tanto primarias como secundarias, se procuró establecer el costo del cumplimiento con el Reglamento de Seguridady Salud en el Trabajo (522-06) para cada una de las empresas participantes en la muestra, estimando el potencial de reducción de accidentes y enfermedades ocupacionales y los ahorros en costos, tanto directos como indirectos, de esta reducción, a los fines de medir el impacto económico y social de un crecimiento en los niveles de prevención de riesgos laborales de este sector. La cuantificación de los costos potenciales fue determinada a partir de las evaluaciones de las empresas y la inversión requerida para el cierre de brechas.

En base a los hallazgos, se determinó un modelo de Programa de Seguridad y Salud para su puesta en práctica en las Pymes, con base en el Reglamento 522-06, así como normativas y estándares internacionales en materia de prevención de accidentes y enfermedades.

A los fines de identificar potenciales fuentes de financiamiento de programas pilotos, así como los planes en materia de prevención de riesgos laborales del Estado, se realizaron entrevistas no estructuradas con personal clave de instituciones gubernamentales con incidencia en el sector.

\subsection{Antecedentes}

El marco teórico para la elaboración de una propuesta de modelo para el desarrollo de programas de seguridad en Pymes puede encontrar en las "Directrices relativas a los sistemas de gestión de la seguridad y la salud en el trabajo ILO-OHS 2001", norma elaborada por la Oficina Internacional del Trabajo (OIT), una guía que garantiza la gestión efectiva de los riesgos y el cumplimiento con los requisitos legales en la materia. Sirvió como base la formulación de los "Términos de Referencia para la Elaboración del Programa de Seguridad y Salud en el Trabajo", elaborados por el Departamento de Monitoreo, Vigilancia y Evaluación de la Dirección General de Higiene y Seguridad Industrial del Ministerio de Trabajo. 
En República Dominicana, hasta enero de 2014 no se registra ningún modelo o investigación relacionado con la implementación de Programas de Seguridad y Salud en las Pymes.

\section{Discusión}

Las estadísticas de accidentes incapacitantes reportados a la Administradora de Riesgos Laborales Salud Segura (ARLSS) (2014) revelan que aunque en el período 2006-2014 ha aumentado el número de casos de 5,359 hasta 30,630, se ha desacelerado la tasa interanual de crecimiento de estos, la cual entre los años 2006 y 2007 fue de 37.77\%, mientras entre los años 2013 y 2014 el incremento fue de $5.48 \%$ y $8.44 \%$, un comportamiento similar al cambio del Producto Interno Bruto (PIB) en igual período, $4.6 \mathrm{y}$ $7.2 \%$, respectivamente, según cifras del Banco Central de la República Dominicana (2015). La referencia a esta correlación viene motivada a que es de esperarse un crecimiento vegetativo del número de casos en función de que aumente el número de inscritos en la Seguridad Social, hecho vinculado con el nivel de actividad económica. Resalta que, por primera vez, desde que se inició la compilación estadística por la ARLSS, el índice de frecuencia de accidentes incapacitantes se redujo, al pasar de 18.66 en 2012 a 18.46 accidentes por cada 200 mil horas hombre trabajadas en el 2013, tomando como base el universo de inscritos en la Tesorería de la Seguridad Social (TSS). En el 2014 fueron 18.55 accidentes/ 200 mil horas, lo que refleja una estabilidad de este indicador en los últimos 3 años (2012-2014), así como en el reporte de casos. 
Identificación de oportunidades para la mejora de la seguridad y salud ocupacional en las Pymes de República Dominicana (primera parte)

\author{
Cuadro N. ${ }^{\circ} 1$
}

Índices de accidentes 2006-2014 y variación respecto al Producto Interno Bruto (PIB)

\begin{tabular}{|c|c|c|c|c|c|c|c|}
\hline Año & $\begin{array}{c}\text { Empleados } \\
\text { sdss }\end{array}$ & Accidentes & $\begin{array}{c}\text { \% } \\
\text { acc }\end{array}$ & $\begin{array}{c}\text { Índice } \\
\text { frec (acc } \\
\mathbf{2 0 0 , 0 0 0} \\
\text { hh) }\end{array}$ & $\begin{array}{c}\text { \% var } \\
\text { acc }\end{array}$ & $\begin{array}{c}\text { \% var } \\
\text { ind frec }\end{array}$ & $\begin{array}{c}\text { \% var } \\
\text { pib }\end{array}$ \\
\hline 2006 & 1.474 .709 & 5.359 & 0,36 & 3,63 & & & \\
\hline 2007 & 1.536 .198 & 7.383 & 0,48 & 4,81 & 37,77 & 32,25 & \\
\hline 2008 & 1.188 .229 & 10.982 & 0,92 & 9,24 & 48,75 & 92,31 & 3,1 \\
\hline 2009 & 1.227 .725 & 14.684 & 1,20 & 11,96 & 33,71 & 29,41 & 0,9 \\
\hline 2010 & 1.299 .087 & 17.456 & 1,34 & 13,44 & 18,88 & 12,35 & 8,3 \\
\hline 2011 & 1.367 .790 & 22.597 & 1,65 & 16,52 & 29,45 & 22,95 & 2,9 \\
\hline 2012 & 1.430 .273 & 26.688 & 1,87 & 18,66 & 18,10 & 12,94 & 2,7 \\
\hline 2013 & 1.530 .322 & 28.246 & 1,85 & 18,46 & 5,84 & $-1,08$ & 4,6 \\
\hline 2014 & 1.651 .202 & 30.630 & 1,86 & 18,55 & 8,44 & 0,50 & 7,2 \\
\hline
\end{tabular}

Fuentes: ARLSS, SISALRIL, Banco Central.

¿Qué factores incidieron en la estabilización de las tasas de crecimiento del porcentaje de accidentes entre 2012 y 2014? El Ministerio de Trabajo ha realizado tres actividades de certificación entre los años 2011 y 2013. En ellas recibieron aprobación de programas por tres años 107 empresas, distribuidas de la siguiente manera: 2001 (40), 2012 (18) y 2013 (49). En el 2014, no hubo certificaciones de programas en ninguna de las dos fechas utilizadas en los años anteriores: el Día Mundial de la Seguridad y Salud en el Trabajo (28 de abril) y el aniversario de la promulgación del Reglamento de Seguridady Salud en el Trabajo (17 de octubre). 
El total de empleados que laboraban en las 107 empresas certificadas hasta el 2013 era de 75,578 personas. Si se amplía el análisis al número de programas presentados entre los años 2010 y 2013, se observa que fueron 280 programas y el número de trabajadores de las mismas 191,690. El cuadro N. ${ }^{\circ} 2$ muestra la distribución de programas presentados por año, donde el promedio de empleados de estas empresas fue de 664, siendo el número más bajo el correspondiente al 440, en promedio en el 2010 y justo un año después fue el número más alto, con promedio de 906 empleados por empresa. Los 191,690 empleados de las 280 empresas representaban el $12.53 \%$ del total de empleados privados registrados en la Seguridad Social hasta ese año (2014). De las empresas privadas que hasta el 2013 fueron certificadas, 87 (83\%) correspondieron a los sectores Hotelería, Comercio e Industrial, y 18 (17\%) a la construcción.

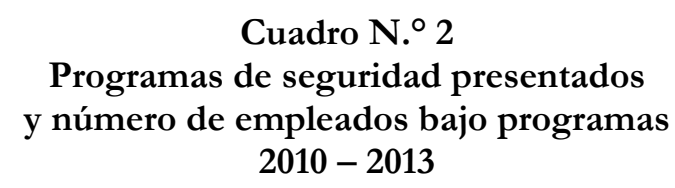

\begin{tabular}{|l|c|c|c|c|c|}
\cline { 2 - 6 } \multicolumn{1}{c|}{} & $\mathbf{2 0 1 0}$ & $\mathbf{2 0 1 1}$ & $\mathbf{2 0 1 2}$ & $\mathbf{2 0 1 3}$ & Total \\
\hline Programas presentados & 27 & 55 & 93 & 105 & 280 \\
\hline Cantidad de trabajadores & 11,890 & 49,825 & 57,653 & 72,322 & 191,690 \\
\hline Promedio de trabajadores & 440 & 906 & 620 & 689 & 664 \\
\hline
\end{tabular}

Fuente: Ministerio de Trabajo.

Según el artículo 1.1 del Reglamento de Seguridady Salud en el Trabajo son las empresas privadas las que deben presentar programas, ya que el mismo define su alcance sobre la base del Código de Trabajo, sin discriminar en su tamaño. El artículo 8.1 del Reglamento de Seguridad y Salud en el Trabajo establece que las empresas deberán presentar sus programas de seguridad y salud al Ministerio de Trabajo dentro de los primeros tres meses de operación. 
El total de empresas privadas registradas en el Seguro de Riesgos Laborales para el año 2014 era de 65,073, con 1, 193,568 empleados. En el universo de empresas inscritas en el seguro $(65,666)$, el $94.67 \%$ tenía menos de 50 empleados. ¿Debería ser el universo de empresas privadas el objetivo de programas a certificar por el Ministerio de Trabajo? ¿Qué estrategias deberán implementarse para incorporar a las Pymes a la mejora de sus condiciones de seguridad y salud? El Reglamento de Riesgos Laborales establece cuatro categorías de riesgos de las empresas, y para diciembre de 2014 el número de empresas por nivel de riesgos estaba distribuido según lo muestra el cuadro N. ${ }^{\circ}$ 3. Las categorías de riesgo las establece el Reglamento de Riesgos Laborales en su artículo 18, definiendo la I para empresas de bajo riesgo, como las oficinas y similares que no utilizan maquinarias y herramientas, dejando las II, III y IV para las demás empresas en función del nivel de peligrosidad. Esto significa que el $41.19 \%$ de las empresas y el 16.5\% de los empleados de República Dominicana laboran en empresas de bajo riesgo.

\section{Cuadro N. ${ }^{\circ} 3$}

Distribución de empresas privadas por categoría de riesgos a diciembre de 2014

\begin{tabular}{|c|c|c|c|c|}
\cline { 2 - 5 } \multicolumn{1}{c|}{} & \multicolumn{2}{c|}{ EMPRESAS } & \multicolumn{2}{c|}{ PORCENTAJE } \\
\hline Categoría & Empresas & Empleados & Empresas & Empleados \\
\hline I & 26.802 & 196.967 & 41,19 & 16,50 \\
\hline II & 16.786 & 429.364 & 25,80 & 35,97 \\
\hline III & 6.063 & 253.746 & 9,32 & 21,26 \\
\hline IV & 15.422 & 313.491 & 23,70 & 26,27 \\
\hline TOTAL & 65.073 & 1.193 .568 & & \multicolumn{1}{c}{} \\
\cline { 1 - 5 } & & \multicolumn{2}{c}{} \\
\cline { 1 - 5 } & &
\end{tabular}

Fuente: SISALRIL. 


\section{Cuadro N. ${ }^{\circ} 4$}

Índice de frecuencia de accidentes incapacitantes por actividad productiva en el año 2013

\begin{tabular}{|c|c|c|c|c|c|c|c|}
\hline $\begin{array}{l}\text { Actividad } \\
\text { Económica }\end{array}$ & \begin{tabular}{|c|} 
Número \\
de \\
Empresas
\end{tabular} & $\begin{array}{c}\% \\
\text { Empr. }\end{array}$ & $\begin{array}{c}\text { Trabajadores } \\
\text { por Sector }\end{array}$ & $\%$ Trab & \begin{tabular}{|c|} 
Total \\
accidentes \\
reportados
\end{tabular} & \begin{tabular}{|c|}
$\%$ \\
Acc/ \\
Sector
\end{tabular} & $\begin{array}{c}\text { IFA (Acc } \\
/ 200,000 \\
\text { hh) }\end{array}$ \\
\hline Pesca & 2 & 0,01 & 16 & 0,00 & 33 & 0,12 & 206,25 \\
\hline Industrias extractivas & 26 & 0,10 & 3733 & 0,33 & 906 & 3,18 & 24,27 \\
\hline Educación & 248 & 0,96 & 19768 & 1,73 & 1688 & 5,92 & 8,54 \\
\hline $\begin{array}{l}\text { Alojamiento y } \\
\text { alimentación }\end{array}$ & 379 & 1,47 & 49519 & 4,34 & 3379 & 11,85 & 6,82 \\
\hline Construcción & 1122 & 4,35 & 31413 & 2,75 & 1516 & 5,32 & 4,83 \\
\hline $\begin{array}{l}\text { Industria de } \\
\text { transformación }\end{array}$ & 1511 & 5,86 & 165818 & 14,52 & 7669 & 26,90 & 4,62 \\
\hline \begin{tabular}{|l|} 
Agricultura, pecuaria \\
y explotación forestal
\end{tabular} & 280 & 1,09 & 13867 & 1,21 & 633 & 2,22 & 4,56 \\
\hline $\begin{array}{l}\text { Producción y distri- } \\
\text { bución de electrici- } \\
\text { dad, gas y agua }\end{array}$ & 109 & 0,42 & 16506 & 1,45 & 695 & 2,44 & 4,21 \\
\hline Servicios domésticos & 4 & 0,02 & 1172 & 0,10 & 41 & 0,14 & 3,50 \\
\hline \begin{tabular}{|l|}
$\begin{array}{l}\text { Salud y servicios } \\
\text { sociales }\end{array}$ \\
\end{tabular} & 737 & 2,86 & 56999 & 4,99 & 1876 & 6,58 & 3,29 \\
\hline \begin{tabular}{|l|} 
Otros servicios \\
colectivos, sociales \\
y personales \\
\end{tabular} & 778 & 3,02 & 26991 & 2,36 & 876 & 3,07 & 3,25 \\
\hline $\begin{array}{l}\text { Comercio y repara- } \\
\text { ción de vehículos } \\
\text { automotrices, } \\
\text { objetos personales } \\
\text { y domésticos } \\
\end{array}$ & 4553 & 17,65 & 150841 & 13,21 & 4622 & 16,21 & 3,06 \\
\hline \begin{tabular}{|l|} 
Transporte, \\
almacenamiento y \\
comunicaciones
\end{tabular} & 622 & 2,41 & 41883 & 3,67 & 1184 & 4,15 & 2,83 \\
\hline $\begin{array}{l}\text { Actividades inmo- } \\
\text { biliarias, avalúos y } \\
\text { servicios prestados } \\
\text { a las empresas } \\
\end{array}$ & 2002 & 7,76 & 57107 & 5,00 & 1603 & 5,62 & 2,81 \\
\hline $\begin{array}{l}\text { Intermediación } \\
\text { financiera }\end{array}$ & 963 & 3,73 & 75345 & 6,60 & 738 & 2,59 & 0,98 \\
\hline \begin{tabular}{|l|} 
Organismos \\
internacionales y \\
otras instituciones \\
extraterritoriales
\end{tabular} & 17 & 0,07 & 553 & 0,05 & 5 & 0,02 & 0,90 \\
\hline \begin{tabular}{|l|} 
Administración \\
pública, defensa y \\
seguridad social
\end{tabular} & 295 & 1,14 & 349989 & 30,65 & 965 & 3,38 & 0,28 \\
\hline Por clasificar & 12147 & 47,09 & 80405 & 7,04 & 84 & 0,29 & 0,10 \\
\hline TOTAL & 25.795 & 100,00 & 1.141 .925 & 100,00 & 28.513 & 100,00 & \\
\hline
\end{tabular}

Fuente: Elaboración propia a partir de datos obtenidos de las Estadísticas de SISALRIL (2014). 

salud ocupacional en las Pymes de República Dominicana (primera parte)

Lentz y Wenz (2006) identificaron los sectores de más altos riesgos y los riesgos más altos para la pequeña y mediana empresa, partiendo del hecho de que los indicadores de fatalidad en las Pymes suelen ser el doble que en las empresas grandes, tanto en Estados Unidos como en Europa.

Según las estadísticas de accidentes del año 2013, suministradas por la Superintendencia de Salud y Riesgos Laborales (SISALRIL), la actividad económica con el mayor índice de frecuencia de accidentes por cada 200,000 horas hombre trabajadas fue la Industria Extractiva, sector donde laboró el $0.24 \%$ de los trabajadores inscritos en la Tesorería de la Seguridad Social (ver cuadro N. $\left.{ }^{\circ} 4\right)$. Le sigue el sector Educación con 8.54, Alojamiento y Alimentación (6.82), Construcción (4.83) y la Industria de Transformación (4.62). Es precisamente en estos tres últimos sectores donde se concentran las empresas que habían presentado sus programas de seguridad hasta el 2013. La pesca, que es el sector con el mayor índice de accidentalidad, solo registró 2 empresas y 16 trabajadores, por lo que para los fines de este análisis no fue considerada esta actividad por su baja representatividad.

Otra estadística que sugiere la necesidad de acción es el índice de accidentes mortales por cada 100,000 afiliados al Seguro de Riesgos Laborales, el cual en el año 2014 dio un salto hasta 13.32 acc / 100 mil afiliados, debido a los 220 fallecidos en el trabajo después de haber alcanzado su nivel más bajo en los últimos 6 años en el 2013, cuando cerró con 10.32 . 
Cuadro N. ${ }^{\circ} 5$

Índice de accidentes mortales

por cada 100,000 trabajadores

2009-2014

\begin{tabular}{|c|c|c|c|}
\hline Año & $\begin{array}{c}\text { Empleados } \\
\text { sdss }\end{array}$ & $\begin{array}{c}\text { Muertes } \\
\text { por accidentes }\end{array}$ & $\begin{array}{c}\text { Tasa mortalidad } \\
\text { (muertes/100,000 } \\
\text { afiliados) }\end{array}$ \\
\hline 2009 & $1,227,725$ & 141 & 11.48 \\
\hline 2010 & $1,299,087$ & 148 & 11.39 \\
\hline 2011 & $1,367,790$ & 201 & 14.70 \\
\hline 2012 & $1,430,273$ & 154 & 10.77 \\
\hline 2013 & $1,530,322$ & 158 & 10.32 \\
\hline 2014 & $1,651,202$ & 220 & 13.32 \\
\hline
\end{tabular}

Fuente: Elaboración propia a partir de datos obtenidos de las Estadísticas de ARLSS (2014).

A pesar de la desaceleración del incremento en el reporte de accidentes en la República Dominicana, el número de empresas que cada año solicitan la reclasificación de su categoría de riesgos es bajo (ver cuadro N. ${ }^{\circ}$ 6), solo 102 entre el 2008 y junio de 2014, siendo 91 a solicitud de las empresas, según estadísticas de la comisión de Riesgos y Tarifas de la Administradora de Riesgos Laborales (ARLSS) (2014), acogiéndose a lo establecido por el Reglamento de Riesgos Laborales, que en sus artículos 19 y 20 da esa prerrogativa a las empresas que reduzcan su siniestralidad en un $25 \%$ de un año a otro. En el mismo período, el número de empresas a las que le fue ajustada hacia arriba la categoría de riesgo fue de 21,409, equivalentes al 99.53\% del total de reclasificadas, fundamentalmente porque la categoría de riesgo no se correspondía con la actividad económica de la empresa. 
Identificación de oportunidades para la mejora de la seguridad y salud ocupacional en las Pymes de República Dominicana (primera parte)

Cuadro N. ${ }^{\circ} 6$

Cambios en las tarifas mensuales otorgadas por el seguro de riesgos laborales

2008-2013

\begin{tabular}{|c|c|c|c|c|c|c|c|c|c|}
\hline Cambios & $\mathbf{2 0 0 8}$ & $\mathbf{2 0 0 9}$ & $\mathbf{2 0 1 0}$ & $\mathbf{2 0 1 1}$ & $\mathbf{2 0 1 2}$ & $\mathbf{2 0 1 3}$ & $\mathbf{2 0 1 4} *$ & Total & $\mathbf{\%}$ \\
\hline Reducción & 17 & 17 & 11 & 32 & 8 & 11 & 6 & 102 & 0,47 \\
\hline Aumento & 1,238 & 2,397 & 5,162 & 5,078 & 4,132 & 2,028 & 1,374 & 21,409 & 99.53 \\
\hline Total & 1,255 & 2,414 & 5,173 & 5,110 & 4,140 & 2,039 & 1,380 & 21,511 & 100.00 \\
\hline
\end{tabular}

Fuente: ARLSS.

El Reglamento de Riesgos Laborales prevé un ajuste en la categoría cuando una empresa sobrepasa el porcentaje asignado a gastos por accidentes y enfermedades ocupacionales. Según el artículo 19, en su acápite d: "Si la tasa de consumo de una empresa originada por la siniestralidad, se encuentra en un rango superior o inferior al $15 \%$ de la contribución de riesgo para prestaciones económicas, la comisión de riesgos y tarifas podrá recomendar a la Superintendencia de Salud y Riesgos Laborales, el ajuste de la contribución complementaria, sujeto a lo establecido en Art. 199 de la Ley 87-01. Este parámetro debe ser obtenido en base a la experiencia real del consumo por la siniestralidad de las empresas".

Pasados diez años del Reglamento de Riesgos Laborales, este artículo sigue sin aplicación, aun cuando el análisis del gasto sugiere que ha habido oportunidad para ello. Por ejemplo, aunque la variación promedio del monto de las prestaciones año tras año entre 2009 y 2013 fue 40.85\% (Prestaciones Económicas ARLSS, 2014), en este último período el cambio fue de solo $14.12 \%$ y el gasto por incapacidad se redujo en $-1.38 \%$ del 2012 al 2013, datos que son congruentes con la reducción de la accidentalidad en el 2013. El número de indemnizaciones y pensiones otorgadas por discapacidad en el período 2008-2013 fue de 1,280 y 431, respectivamente. 


\section{Cuadro N. ${ }^{\circ} 7$}

Porcentaje de variación interanual por categoría del gasto 2008-2013

\begin{tabular}{|c|c|c|c|c|}
\hline Año & $\begin{array}{c}\mathbf{\%} \\
\text { Prestaciones }\end{array}$ & $\begin{array}{c}\mathbf{\%} \\
\text { Incapacidades }\end{array}$ & $\begin{array}{c}\mathbf{\%} \\
\text { Indemnizaciones }\end{array}$ & $\begin{array}{c}\mathbf{\%} \\
\text { Pensiones }\end{array}$ \\
\hline 2009 & 23.74 & 38.20 & 6.97 & -3.90 \\
\hline 2010 & 39.95 & 16.13 & -7.96 & 26.08 \\
\hline 2011 & 89.00 & 51.11 & -1.74 & 52.78 \\
\hline 2012 & 37.44 & 31.79 & 33.07 & 14.80 \\
\hline 2013 & 14.12 & -1.38 & 102.15 & 32.36 \\
\hline PROM & 40.85 & 27.17 & 26.50 & 24.42 \\
\hline
\end{tabular}

Fuente: Elaboración propia a partir de datos obtenidos de las Prestaciones Económicas ARLSS 2014.

Brosseau y Li (2005) aplicaron una encuesta a 4084, empresas manufactureras con un número de empleados entre 5 y 50 de la ciudad de Minnesota, Estados Unidos, explorando las variables que subyacen en las intenciones de mejorar la seguridad y la salud en el trabajo por parte de propietarios de pequeños negocios, concluyendo que los resultados de la empresa, las normativas y la creencia en los controles son los factores que priman en su actitud hacia la mejora de las condiciones de trabajo. En un contexto en el que el Ministerio de Trabajo tiene una baja capacidad de coerción, el empresario dominicano carece de incentivos a la mejora de su desempeño en seguridad, como lo debería ser la reducción de las primas de seguros. Al mes de abril de 2014, la Dirección General de Higiene y Seguridad Industrial del Ministerio de Trabajo contaba con 24 empleados, distribuidos según lo detalla el cuadro N. ${ }^{\circ} 8$. 
Identificación de oportunidades para la mejora de la seguridad y salud ocupacional en las Pymes de República Dominicana (primera parte)

\section{Cuadro N. ${ }^{\circ} 8$}

Personal Dirección General de Higiene y Seguridad Industrial, Ministerio de Trabajo

\begin{tabular}{|c|c|c|c|c|c|}
\cline { 2 - 6 } \multicolumn{1}{c|}{} & \multicolumn{5}{c|}{ Localidad } \\
\hline Función & $\begin{array}{c}\text { Santo } \\
\text { Domingo }\end{array}$ & $\begin{array}{c}\text { La } \\
\text { Romana }\end{array}$ & Santiago & $\begin{array}{c}\text { Puerto } \\
\text { Plata }\end{array}$ & Higuey \\
\hline Evaluación & 14 & 1 & 3 & 1 & 1 \\
\hline Analistas de S y S & & 3 & 1 & & \\
\hline $\begin{array}{c}\text { Asistente } \\
\text { Administrativa }\end{array}$ & & & & & \\
\hline $\begin{array}{c}\text { Divulgación } \\
\text { y Promoción }\end{array}$ & 3 & & & & \\
\hline
\end{tabular}

Fuente: Ministerio de Trabajo de República Dominicana.

Entre los años 2007 y 2013 fueron registrados en el Ministerio de Trabajo 3184 Comités de Seguridad y Salud en el Trabajo, de los cuales, para junio de 2014, solo 1235 (39\%) se encontraban activos es decir, remitiendo a dicha entidad las minutas de reuniones mensuales de forma regular, según lo establecido en el artículo 6.4.2 del Reglamento de Seguridad y Salud en el Trabajo. Para mejorar la situación, la Dirección General de Higiene y Seguridad Industrial instituyó desde febrero de 2012 la Escuela de Comités de Seguridad, donde participaron, a partir de dicha fecha hasta diciembre de 2013, unas 2,817 personas. (Fuente: Ministerio de Trabajo, año 2014).

La función asesora del Ministerio de Trabajo, en materia de seguridad, es propia de los organismos reguladores, con independencia a su esfuerzo coercitivo. Por ejemplo, según Greife (2001), en Estados Unidos, la Administración de Seguridad y Salud Ocupacional (OSHA) cuenta con un equipo de consultores que, con base a la 29 CFR Part. 1908, asesoran pequeñas empresas de sectores o actividades de alto riesgo, en un programa voluntario que genera reportes confidenciales (no se genera persecución a 
partir de deficiencias encontradas) y cuyo único compromiso del empleador (en su mayoría empresas de menos de 10 empleados) es la implementación de las recomendaciones.

En lo relativo al brazo coercitivo del Ministerio de Trabajo para incrementar el cumplimiento del Reglamento de Seguridad y Salud en el Trabajo, el mismo se ejerce a través de los inspectores de las 40 Representaciones Locales de Trabajo (RLT), labor para la cual en junio de 2014, según la "Relación de las Empresas Con Violación Al Reglamento 522-06, Sobre Seguridad y Salud en el Trabajo" existían 189 inspectores distribuidos en todo el territorio nacional, 37.04\% de los cuales se concentraban en el Gran Santo Domingo (19.58\% Distrito Nacional, $11.11 \%$ en Santo Domingo Este y 6.35\% en Santo Domingo Oeste). Santiago de los Caballeros tenía 7.94\%, San Pedro de Macorís 4.76\% y La Romana 3.7\%, para totalizar 53.44\%.

El esfuerzo de este equipo dio como resultado que en el 2013 se realizaran 69,949 inspecciones, de las cuales 55,304 fueron regulares $(79.1 \%)$ y 14,645 especiales $(20.9 \%)$. De estas inspecciones se derivaron 131 infracciones relacionadas con violaciones al Reglamento de Seguridad y Salud en el Trabajo. Ellas representaron el $2.7 \%$ del total de infracciones levantadas durante el año. El cuadro N. 9 muestra la proporción desde el año 2009 al 2013, donde después de un año record de 151 infracciones, los siguientes dos años las proporciones cayeron 1.6 y $0.6 \%$, respectivamente, como un total del número de infracciones levantadas por el Sistema de Inspección del Ministerio de Trabajo.

\section{Cuadro N. ${ }^{\circ} 9$}

Infracciones relacionadas con violaciones al

Reglamento de Seguridad y Salud en el Trabajo

\begin{tabular}{|c|c|c|c|c|c|}
\hline Infracciones & $\mathbf{2 0 0 9}$ & $\mathbf{2 0 1 0}$ & $\mathbf{2 0 1 1}$ & $\mathbf{2 0 1 2}$ & $\mathbf{2 0 1 3}$ \\
\hline Reglamento SST & 151 & 60 & 20 & 125 & 131 \\
\hline \% Total & 4.4 & 1.6 & 0.6 & 3.3 & 2.7 \\
\hline
\end{tabular}

Fuente: Dirección de Coordinación del Sistema de Inspección, formulario RLT-03. 
Si se observa el cuadro N. ${ }^{\circ} 10$, se podrá notar cómo en el período de 2009-2013 el número de inspecciones se redujo desde 88,317, en el 2009 a 69,949, en el 2013.

\author{
Cuadro N. ${ }^{\circ} 10$
}

Visitas de inspección del trabajo por tipo de actividad 2009-2013

\begin{tabular}{|c|c|c|c|c|c|}
\hline Inspecciones & $\mathbf{2 0 0 9}$ & $\mathbf{2 0 1 0}$ & $\mathbf{2 0 1 1}$ & $\mathbf{2 0 1 2}$ & $\mathbf{2 0 1 3}$ \\
\hline Regulares & 63,697 & 65,487 & 56,010 & 52,261 & 55,304 \\
\hline Especiales & 24,620 & 20,665 & 16,773 & 15,989 & 14,645 \\
\hline Total & 88,317 & 86,152 & 72,783 & 68,250 & 69,949 \\
\hline
\end{tabular}

Fuente: Ministerio de Trabajo.

El monto asignado en el Presupuesto Nacional al Ministerio de Trabajo entre los años 2008 y 2011 tuvo apenas un incremento de 30 millones de pesos (ver cuadro N. ${ }^{\circ} 11$ ), lo que limitó la adaptación de la Dirección General de Higiene y Seguridad Industrial a las capacidades requeridas para dar seguimiento al nuevo reglamento surgido en el 2006. Hasta dicho año, la división responsable de la puesta en práctica del Reglamento de Seguridad y Salud en el Trabajo mantuvo su mismo número de empleados (6) al año previo a la emisión del mismo.

Entre enero y mayo de 2014 fueron sometidas 11 empresas, 7 de las cuales se relacionaron con la falta de Comité de Seguridad y 3 con Seguridad en la Construcción (Ministerio de Trabajo, 2014). Sin embargo es evidente un enfoque sesgado: 4 de las 7 empresas sometidas por el artículo 6.1 (Comité de Seguridad) fueron compañías de vigilancia, mientras las 3 violaciones del sector construcción fueron a la misma empresa (capítulo 2.3). Otro tema que sale reflejado en los sometimientos es la inconsistencia en la numeración del Reglamento de Seguridady Salud en el Trabajo, generándose confusión entre lo que es un capítulo, una sección o un artículo. 


\section{Cuadro N. ${ }^{\circ} 11$ \\ Presupuesto del Ministerio de Trabajo y su ejecución durante el período 2007-2013}

\begin{tabular}{|c|c|c|c|c|c|c|}
\hline Presup. & $\begin{array}{c}\text { Original } \\
\mathbf{( 2 )}\end{array}$ & $\begin{array}{c}\text { Modificación } \\
\mathbf{( 3 )}\end{array}$ & $\begin{array}{c}\text { Vigente } \\
\mathbf{( 4 )}\end{array}$ & $\begin{array}{c}\text { Ejecutado } \\
\mathbf{( 1 0 )}\end{array}$ & $\begin{array}{c}\text { Var Pres } \\
\text { anual } \\
\mathbf{( \% )}\end{array}$ & $\begin{array}{c}\text { Var } \\
\text { Ejecución } \\
\text { Anual (\%) }\end{array}$ \\
\hline $\mathbf{2 0 0 7}$ & $810,106,053$ & $207,725,350$ & $1,017,831,403$ & $958,068,861$ & & \\
\hline $\mathbf{2 0 0 8}$ & $1,448,333,362$ & $-6.520,381$ & $1,441,812,981$ & $1,240,348,869$ & 78.78 & 29.46 \\
\hline $\mathbf{2 0 0 9}$ & $1,433,858,189$ & $147,721,509$ & $1.581,579,698$ & $1,451,899,855$ & -1.00 & 17.06 \\
\hline $\mathbf{2 0 1 0}$ & 1.484 .266 .564 & $-361,081,456$ & $1,123,185,108$ & $1,081,369,498$ & 3.52 & -25.52 \\
\hline $\mathbf{2 0 1 1}$ & $1,478.461,043$ & $-65,097,135$ & $1,413,363,908$ & $1,265,404 ., 71$ & -0.39 & 17.02 \\
\hline $\mathbf{2 0 1 2}$ & $1,689,560,021$ & $32,454,559$ & $1,722,014,580$ & $1,657,766,517$ & 14.28 & 31.01 \\
\hline $\mathbf{2 0 1 3}$ & $1,738,109,956$ & $67,685,600$ & $1,805,795,556$ & $1,651,820,017$ & 2.87 & -0.36 \\
\hline
\end{tabular}

Fuente: Ministerio de Trabajo.

Debe señalarse que, por la naturaleza del trabajo de las compañías de seguridad privada, los comités de seguridad les son poco funcionales, dado que los guardianes están dispersos en las organizaciones a las que prestan servicios. Para obtener una perspectiva respecto a los sometimientos, vale revisar un artículo de opinión escrito por el licenciado Luis Manuel Vílchez Bournigal, titulado "Nuevas maneras de hostigar a las Pymes", el cual describe la situación desde el punto de vista de un abogado laboral: "El nuevo mecanismo de acoso del Ministerio de Trabajo y de sus inspectores consiste en levantar actas de apercibimiento en contra de aquellas empresas que no observan el Art. 6 de la resolución 04-07 del Reglamento No. 522-06 sobre Seguridad y Salud en el Trabajo, es decir empresas con más de quince (15) empleados que no han conformado un comité mixto de Seguridad y Salud en el Trabajo" (Vílchez, 2014). 

salud ocupacional en las Pymes de República Dominicana (primera parte)

El autor del artículo también cuestiona el costo que para las pequeñas y medianas empresas tiene la presentación de un programa de seguridad y salud, según lo establece el artículo 8.1 del Reglamento de Seguridady Salud en el Trabajo, y a continuación ofrece un dato sobre una jurisprudencia establecida por la Suprema Corte de Justicia (SCJ), afirmando que la sentencia dictada por la Tercera Sala de la SCJ, de fecha 27 de abril de 2012, B. J. 1217, señalando que "la falta de remitir al Ministerio de Trabajo un programa de seguridad y salud en el trabajo no es una falta grave cuando se logra comprobar que la empresa está cumpliendo con las leyes relativas a la seguridad social, en virtud de que el derecho de trabajo no se nutre de documentos, sino de realidades".

Una lectura de la sentencia completa evidencia un hecho citado en entrevista a Vílchez y Parra (2014): la no presentación de programas de seguridad según lo consigna el artículo 8.1 del Reglamento de Seguridady Salud en el Trabajo está siendo usado como causa de dimisión por trabajadores que llevan a sus empleadores a juicio, sin que necesariamente prime el interés en ellos de mejorar las condiciones del lugar de trabajo. Las asociaciones empresariales proponen que se viabilicen formas de cumplimiento del Reglamento de Seguridady Salud para las Pymes.

En Estados Unidos, el Instituto Nacional para la Seguridad y Salud Ocupacional (NIOSH) desarrolló un sitio web para el apoyo a la prevención de riesgos laborales en pequeños negocios, sin la necesidad de invertir mucho tiempo y dinero (Professional Safety, 2013). Uno de los recursos que ofrece, la Guía para el Plan de Prevención de Accidentes, recomienda 8 componentes (administración, registros, análisis de seguridad, prevención de accidentes, reporte e investigación de accidentes, entrenamiento, inspecciones de seguridad y revisión del programa), lo cual contrasta con los 20 elementos que requiere el Capítulo 7 del Reglamento de Seguridad y Salud en el Trabajo, que no discrimina el requerimiento según tipo ni tamaño de las empresas (Ver cuadro N. $\left.{ }^{\circ} 11\right)$. Esta guía es 
provista por el Departamento de Seguros de Texas, y en general el sitio web se nutre de colaboraciones (NIOSH, 2014).

En Irlanda, donde los negocios con menos de 50 empleados son el 95\% del total país (Health and Safety Administration, 2014), la Autoridad de Seguridad y Salud desarrolla a partir de 2007 un conjunto de herramientas para la mejora de la seguridad, la salud y el bienestar en los pequeños negocios, con enfoque al cumplimiento legal (Veterinary Ireland Journal, 2011). Allí, las regulaciones en la materia no excluyen empresas por sector ni tamaño, pues consideran que ningún negocio es tan pequeño para no recibir los beneficios de una efectiva gestión de la seguridad y la salud.

En definitiva, el aspecto económico es un factor que limita la implementación de las medidas de prevención; sin embargo, no es el único. Kheni, Gibb y Dainty (2010) analizaron la seguridad y la salud en la construcción en Ghana, un sector con alta presencia de Pymes que contribuía con el $8.8 \%$ de Producto Interno Bruto y empleaba el 1.8\% de la población económicamente activa de dicho país, según datos del Fondo Monetario Internacional del 2005.

En su examen de la literatura previa, concluyen que la principal prioridad de las Pymes es la supervivencia, por lo que la inversión en seguridad y salud queda en un plano secundario. El estudio identificó entre las principales limitaciones que afectan la prevención en las Pymes dedicadas a la construcción en Ghana: el bajo nivel educativo y social de los trabajadores, el desconocimiento de sus dueños sobre sus responsabilidades en la materia y una inefectiva gestión de las autoridades de seguridad y salud, abogando por una más proactiva gestión de la prevención de riesgos laborales que tome en cuenta la cultura de trabajo de las Pymes. 
Identificación de oportunidades para la mejora de la seguridad y salud ocupacional en las Pymes de República Dominicana (primera parte)

\section{Cuadro N. ${ }^{\circ} 12$}

Comparación de requerimientos entre programas de seguridad y salud del reglamento 522-06 y las recomendaciones de la guía del plan de prevención de accidentes de NIOSH

\begin{tabular}{|c|c|}
\hline $\begin{array}{l}\text { Requerimientos para Programas de } \\
\text { Seguridad y Salud del Reglamento } \\
\text { de Seguridad y Salud en el Trabajo }\end{array}$ & $\begin{array}{l}\text { Componentes de la Guía del Plan de } \\
\text { Prevención de Accidentes de NIOSH }\end{array}$ \\
\hline \multicolumn{2}{|l|}{ 1. Análisis de Accidentes/Incidentes } \\
\hline \multicolumn{2}{|l|}{ 2. Comunicaciones personales } \\
\hline \multicolumn{2}{|l|}{ 3. Contratación y colocación } \\
\hline \multicolumn{2}{|l|}{ 4. Control de salud } \\
\hline \multicolumn{2}{|l|}{ 5. Controles de compras } \\
\hline \multicolumn{2}{|l|}{ 6. Controles de Ingeniería } \\
\hline 7. Entrenamiento de Empleo & \multirow{2}{*}{ 6.- Entrenamiento } \\
\hline 8. Entrenamiento de la Administración & \\
\hline \multicolumn{2}{|l|}{ 9. Equipo de Protección Personal } \\
\hline 10. Inspecciones Planeadas & 7.- Inspecciones de seguridad \\
\hline 11. Investigación de accidentes/incidentes & 5.- Reporte e investigación de accidentes \\
\hline 12. Liderazgo y Administración & 1.- Administración \\
\hline \multicolumn{2}{|l|}{ 13. Observaciones de tareas } \\
\hline \multicolumn{2}{|l|}{ 14. Preparación para emergencias } \\
\hline 15. Procedimientos y Análisis de tareas & 3.- Análisis de seguridad \\
\hline \multicolumn{2}{|l|}{ 16. Promoción General } \\
\hline 17. Reglas de la organización & 4.- Prevención de accidentes \\
\hline \multicolumn{2}{|l|}{ 18. Reuniones de grupo } \\
\hline \multicolumn{2}{|l|}{ 19. Seguridad Fuera del Trabajo } \\
\hline \multirow[t]{2}{*}{ 20. Sistema de evaluación del programa } & 8.- Revisión del programa \\
\hline & 2.- Registros \\
\hline
\end{tabular}

Fuente: Elaboración propia a partir de datos obtenidos del Reglamento de seguridady salud en el Trabajo y recomendaciones de la guía del plan de prevención de accidentes de NIOSH. 
Investigaciones hechas por estudiantes de las Maestrías de Prevención de Riesgos Laborales y Administración de la Construcción del INTEC, en los trimestres mayo-julio (Maestría de Prevención de Riesgos Laborales) y agosto-octubre (Maestría de Administración de la Construcción) de 2014, exploraron en torno al cumplimiento del Reglamento de Seguridad y Salud en las Pymes de República Dominicana y el costo de formulación y posterior implementación de los Programas de Seguridad y Salud en las mismas.

Una muestra de 20 empresas de entre 15 y 200 empleados, representativas de 15 de las actividades económicas en las cuales subdivide la ARLSS los accidentes, arrojó como resultado que el nivel de cumplimiento con el Reglamento de Seguridad y Salud en el Trabajo fue de $50.65 \%$ en promedio y un costo estimado de $\$ 742,495.69$ para el desarrollo e implementación de los Programas de Seguridad y Salud en el trabajo. De las 20 empresas, 5 pertenecían a la industria de transformación, donde el nivel de cumplimiento fue de $60.8 \%$ y el costo de implementación $\$ 420,820.57$.

El otro sector con evaluaciones múltiples fue el comercial (3 empresas). Allí el cumplimiento encontrado fue de $64 \%$ y el costo de implementación proyectado $\$ 398,377.51$. Para 2 constructoras evaluadas, el promedio de cumplimiento fue $41 \%$ y el costo de implementación del programa de seguridad y salud en ellas, de acuerdo a la demanda de cierre de brechas de cumplimiento con el Reglamento de Seguridad y Salud en el Trabajo (522-06), fue de $\$ 2,102,041.47$. Para las restantes 10 empresas Pymes (forestal, pesca, mina, servicios, restaurante, sucursal bancaria, educación, salud, inmobiliaria, almacenamiento), el promedio de cumplimiento fue $49.3 \%$ y el costo proyectado de implementación del programa $\$ 672,420.45$. 
Identificación de oportunidades para la mejora de la seguridad y salud ocupacional en las Pymes de República Dominicana (primera parte)

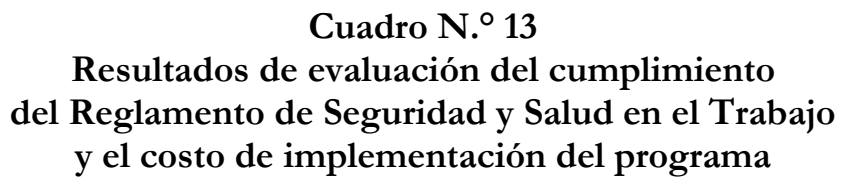

\begin{tabular}{|l|c|c|c|}
\hline \multicolumn{1}{|c|}{ Sector } & Muestras & $\begin{array}{c}\text { \% } \\
\text { Cumplimiento }\end{array}$ & $\begin{array}{c}\text { Costo } \\
\text { implementación (\$) }\end{array}$ \\
\hline $\begin{array}{l}\text { Industria de } \\
\text { Transformación }\end{array}$ & 5 & 50.65 & $420,820.57$ \\
\hline Comercial & 3 & 64.00 & $398,377.51$ \\
\hline Construcción & 2 & 41.00 & $2,102,041.47$ \\
\hline Otros & 10 & 49.30 & $\mathbf{6 7 2 , 4 2 0 . 4 5}$ \\
\hline General (Promedio) & $\mathbf{2 0}$ & $\mathbf{5 0 . 6 5}$ & $\mathbf{7 4 2 , 4 9 5 . 6 9}$ \\
\hline
\end{tabular}

Fuente: Elaboración propia a partir de datos obtenidos de la Evaluación del cumplimiento del Reglamento de Seguridad y Salud en el Trabajo y el costo de implementación del programa.

Vale resaltar que los costos de implementación se verán influenciados por el número de empleados y el tipo de industria. En el primer factor, los equipos de protección y las pruebas médicas deberán considerar los riesgos laborales: la probabilidad de una afección respiratoria requerirá el uso de mascarillas y la evaluación de la función pulmonar. De ahí que, a mayor nivel de riesgo de la industria, mayores costos de implementación, y es allí donde el derecho a la protección de que habla el Reglamento de Seguridad y Salud en el Trabajo choca con las capacidades de cumplimiento que tienen las Pymes con dicho deber. Surge la pregunta ¿Deberá depender el derecho a la protección del trabajador del tamaño de la empresa o de su nivel de riesgos? 


\section{Conclusiones}

a) Aunque el 95\% de las empresas dominicanas tiene menos de 50 empleados, el Reglamento de Seguridad y Salud en el Trabajo (522-06) no distingue nivel de exigencias por tamaño o nivel de riesgos para los Programas de Seguridad y Salud, variables que son consideradas en otros países para adaptar los requisitos regulatorios a las condiciones de las Pymes.

b) El análisis de las tasas de accidentalidad de la República Dominicana evidencia la existencia de sectores prioritarios para el trabajo de promoción de la prevención, como son los de educación y alojamiento y alimentación, los cuales tienen índices mayores a otros como el de construcción y transformación, que son señalados con frecuencia como de más alto riesgo.

c) A pesar de que el Reglamento de Riesgos Laborales establece incentivos para las empresas que mejoran su desempeño en materia de Seguridad y Salud, o tienen bajo nivel de reclamos, los mismos no son aplicados por la Administradora de Riesgos Laborales (ARLSS).

d) El Ministerio de Trabajo y su Dirección General de Higiene y Seguridad Industrial carecen de recursos suficientes para vigilar el cumplimiento del Reglamento de Seguridad y Salud en el Trabajo (522-06). Salvo su Escuela de Comités de Seguridad, no disponen de herramientas de apoyo a las empresas que lo requieren para la implementación de sus programas.

e) El esfuerzo del Ministerio de Trabajo en procura de hacer cumplir el Reglamento de Seguridad y Salud en el Trabajo (522-06) ha sido sesgado, además de limitado, sin criterios técnicos claros para justificar el enfoque en los sectores elegidos, como es el de la construcción. 
Identificación de oportunidades para la mejora de la seguridad y salud ocupacional en las Pymes de República Dominicana (primera parte)

\section{Recomendaciones y propuestas}

a) Desarrollar programas específicos para la prevención de accidentes en sectores con alta accidentalidad, como son los de educación y alojamiento y alimentación, según estadísticas mostradas en el cuadro N. ${ }^{\circ} 4$.

b) Fortalecer la función asesora del Ministerio de Trabajo y su Dirección General de Higiene y Seguridad Industrial, mediante la puesta a disposición de las Pymes de consultores dedicados de forma exclusiva a brindar soporte a la formulación e implementación de Programas de Seguridad y Salud en sectores seleccionados, como puede ser el de la construcción, donde la Dirección General de Higiene y Seguridad Industrial ha mostrado mucho interés, con metas específicas e indicadores de desempeño para la iniciativa. (Ver en el anexo 1 un modelo de la propuesta).

c) Capacitar a los inspectores de trabajo para que estos, en sus visitas a las empresas, incluyan la vigilancia al cumplimiento del Reglamento de Seguridady Salud en el Trabajo (522-06). El propósito es expandir el conocimiento del Reglamento por medio del apercibimiento. El esfuerzo puede ir acompañado de un levantamiento del estado actual del cumplimiento del Reglamento, de forma que las 70,000 visitas que estos realizan en promedio por año sirvan en un esfuerzo de doble vía: educativo y diagnóstico.

d) Simplificar los requisitos de los Programas de Seguridad y Salud para las Pymes (ver anexo 2), y crear pilotos para la formulación de programas y el seguimiento a los resultados de la implementación, midiendo la incidencia que dichos esfuerzos produzcan con relación a la mejora de las condiciones de trabajo en los sectores seleccionados. 


\section{Anexos}

\subsection{Anexo I}

\section{Metas e indicadores de programas pilotos sectoriales del ministerio de trabajo}

\begin{tabular}{|c|c|c|c|}
\hline $\mathbf{N} .^{\circ}$ & Objetivo & Meta & Indicadores \\
\hline 1 & $\begin{array}{l}\text { Aumentar el número de } \\
\text { Planes de Seguridad en } \\
\text { Obras que presentan las } \\
\text { construcciones previo a } \\
\text { su inicio. }\end{array}$ & $\begin{array}{l}\text { Aumentar el número de Planes } \\
\text { de Seguridad en Obras en un } \\
50 \% \text { con respecto al año an- } \\
\text { terior. }\end{array}$ & $\begin{array}{l}\text { Porcentaje de obras } \\
\text { que presentan planes } \\
\text { de seguridad previo a } \\
\text { su inicio. }\end{array}$ \\
\hline 2 & $\begin{array}{l}\text { Reducir la accidentalidad } \\
\text { del sector construcción } \\
\text { en las empresas registra- } \\
\text { das en la TSS. }\end{array}$ & $\begin{array}{l}\text { Reducir en un } 25 \% \text { la acci- } \\
\text { dentalidad del sector cons- } \\
\text { trucción, en base a reportes } \\
\text { a la ARLSS. }\end{array}$ & $\begin{array}{l}\text { Índice de accidentalidad } \\
\text { del sector. }\end{array}$ \\
\hline 3 & $\begin{array}{l}\text { Aumentar el cumpli- } \\
\text { miento del capítulo } \\
\text { Construcción del Regla- } \\
\text { mento de Seguridady Salud } \\
\text { en el Trabajo. }\end{array}$ & $\begin{array}{l}\text { Llevar el nivel de cumpli- } \\
\text { miento del Listado de Seguri- } \\
\text { dad en la Construcción a un } \\
\text { mínimo promedio de } 80 \% \\
\text { entre las empresas que for- } \\
\text { man parte del programa. }\end{array}$ & $\begin{array}{l}\text { Porcentaje de cumpli- } \\
\text { miento del Listado de } \\
\text { Seguridad en Obras. }\end{array}$ \\
\hline 4 & $\begin{array}{l}\text { Expandir a nivel nacional } \\
\text { el conocimiento del } \\
\text { Capítulo } 3 \text { del Reglamento } \\
\text { de Seguridady Salud en el } \\
\text { Trabajo. }\end{array}$ & $\begin{array}{l}\text { Lograr que para el 1er año del } \\
\text { programa los Planes de Segu- } \\
\text { ridad en Obras presentados } \\
\text { por construcciones fuera del } \\
\text { Distrito Nacional representen } \\
\text { el } 20 \% \text { del total de Planes de } \\
\text { Seguridad en Obras. }\end{array}$ & $\begin{array}{l}\text { Número de planes de } \\
\text { seguridad presentados } \\
\text { por regiones. }\end{array}$ \\
\hline 5 & $\begin{array}{l}\text { Desarrollar programas de } \\
\text { capacitación en seguridad } \\
\text { en obras para el sector } \\
\text { construcciones en locali- } \\
\text { dades seleccionadas. }\end{array}$ & $\begin{array}{l}\text { Capacitar al menos 1,000 } \\
\text { trabajadores del sector } \\
\text { construcción en los aspectos } \\
\text { que están regulados del sector. }\end{array}$ & $\begin{array}{l}\text { Número de personas } \\
\text { capacitadas en seguri- } \\
\text { dad en la construcción. }\end{array}$ \\
\hline
\end{tabular}


Identificación de oportunidades para la mejora de la seguridad y salud ocupacional en las Pymes de República Dominicana (primera parte)

\subsection{Anexo II}

\section{Elementos del programa de seguridad y salud para Pymes y su correlación con los 20 elementos y la guía del plan de prevención NIOSH}

\begin{tabular}{|c|c|c|}
\hline $\begin{array}{l}\text { Requerimientos para programas } \\
\text { de seguridad y salud del } \\
\text { reglamento de seguridad } \\
\text { y salud en el trabajo }\end{array}$ & $\begin{array}{l}\text { Componentes de la } \\
\text { guía del plan de } \\
\text { prevención de acci- } \\
\text { dentes de NIOSH }\end{array}$ & $\begin{array}{c}\text { Propuesta de } \\
\text { programa de seguridad } \\
\text { y salud para Pymes }\end{array}$ \\
\hline \multicolumn{3}{|l|}{ 1. Análisis de accidentes/incidentes } \\
\hline 2. Comunicaciones personales & & Inducción en seguridad \\
\hline \multicolumn{3}{|l|}{ 3. Contratación y colocación } \\
\hline \multicolumn{3}{|l|}{ 4. Control de salud } \\
\hline 5. Controles de compras & & $\begin{array}{l}\text { Información de seguridad } \\
\text { de materiales }\end{array}$ \\
\hline \multicolumn{3}{|l|}{ 6. Controles de ingeniería } \\
\hline 7. Entrenamiento de empleo & \multirow{2}{*}{ Entrenamiento } & \multirow{2}{*}{ Entrenamiento } \\
\hline 8. Entrenamiento de la administración & & \\
\hline 9. Equipo de protección personal & & $\begin{array}{l}\text { Protección personal y } \\
\text { colectiva }\end{array}$ \\
\hline 10. Inspecciones planeadas & $\begin{array}{l}\begin{array}{l}\text { Inspecciones de } \\
\text { seguridad }\end{array} \\
\end{array}$ & $\begin{array}{l}\text { Inspecciones de } \\
\text { seguridad }\end{array}$ \\
\hline $\begin{array}{l}\text { 11. Investigación de accidentes/ } \\
\text { incidentes }\end{array}$ & $\begin{array}{l}\text { Reporte e investiga- } \\
\text { ción de accidentes }\end{array}$ & $\begin{array}{l}\text { Reporte e investigación } \\
\text { de accidentes }\end{array}$ \\
\hline 12. Liderazgos y administración & Administración & Política de seguridad \\
\hline \multicolumn{3}{|l|}{ 13. Observaciones de tareas } \\
\hline 14. Preparación para emergencias & & $\begin{array}{l}\text { Preparación para } \\
\text { emergencias }\end{array}$ \\
\hline $\begin{array}{l}\text { 15. Procedimientos y análisis de } \\
\text { tareas }\end{array}$ & Análisis de seguridad & $\begin{array}{l}\text { Evaluación y control de } \\
\text { riesgos }\end{array}$ \\
\hline \multicolumn{3}{|l|}{ 16. Promoción general } \\
\hline 17. Reglas de la organización & $\begin{array}{l}\text { Prevención de acci- } \\
\text { dentes }\end{array}$ & $\begin{array}{l}\text { Reglamento interno de } \\
\text { seguridad }\end{array}$ \\
\hline 18. Reuniones de grupo & & Comité de seguridad \\
\hline \multicolumn{3}{|l|}{ 19. Seguridad fuera del trabajo } \\
\hline \multirow[t]{2}{*}{$\begin{array}{l}\text { 20. Sistema de evaluación del } \\
\text { programa }\end{array}$} & Revisión del programa & Revisión del programa \\
\hline & Registros & $\begin{array}{l}\text { Documentación y } \\
\text { registros }\end{array}$ \\
\hline
\end{tabular}




\section{Referencias}

Administradora de Riesgos Laborales Salud Segura. (2014). Estadísticas. Recuperado de www.arlss.gov.do

Administradora de Riesgos Laborales Salud Segura. (2014). Empresas Reclasificadas Período 2008-2013. Santo Domingo: ARLSS.

Administradora de Riesgos Laborales Salud Segura. (2014). Prestaciones Económicas 2008-2013. Santo Domingo: ARLSS.

Banco Central de la República Dominicana. (2015). Producto Interno Bruto 2007-2014. Recuperado de www.bancentral.gov.do

Brosseau, 1. \& Lishelby, Y. (2005). Small business owners' health and safety intentions: A cross-sectional survey. Environmental Health: A Global Access Science Source, 4, 9-23.

Greife, A. (2001). Surveillance of Safety and Health Programs and Needs in Small U.S. Businesses. Applied Occupational and Environmental Hygiene, 16(11), 1016-1021.

Health and Safety Administration. (2014). Página de inicio. Recuperado de http://www.hsa.ie/eng/Small_Business/Getting_ Started/

Health and Safety in Small Businesses. Veterinary Ireland Journal, 64(2), 63-90.

Kheni, N.; Gibb, A. \& Dainty, A. (2010). Health and Safety Management within Small- and Medium-Sized Enterprises (SMEs) in Developing Countries: Study of Contextual Influences. Journal of Construction Engineering and Management, 1104-1115.

Lentz, T. \& Wenzl, T. (2006). Small Businesses with High Fatality Rates: Assessment of Hazards and Their Prevention. Journal of Occupational \& Environmental Hygiene, 3(2), 8-14. 

salud ocupacional en las Pymes de República Dominicana (primera parte)

National Institute of Occupational Safety and Health. (2014). Small Business Resource Guide. Recuperado de http://www.cdc.gov/ niosh/topics/smbus/guide/guide-2.html

Professional Safety. (2013). NIOSH Offers Safety Guidance to Small Businesses. Professional Safety, 58(5), 20-22.

República Dominicana. (2007). Reglamento de Seguridady Salud en el Trabajo. Santo Domingo: Ministerio de Trabajo.

República Dominicana. (2013). Reglamento de Riesgos Laborales. Santo Domingo: SISALRIL.

República Dominicana. (2014). Estadísticas de Programas de Seguridady Salud Presentados. Dirección General de Higiene y Seguridad Industrial. Santo Domingo: Ministerio de Trabajo.

República Dominicana. (2014). Estadísticas de Comités de Seguridad. Dirección General de Higiene y Seguridad Industrial. Santo Domingo: Ministerio de Trabajo.

República Dominicana. (2014). Estadísticas de Inspecciones de Trabajo. Dirección de Coordinación del Sistema de Inspección. Santo Domingo: Ministerio de Trabajo.

República Dominicana. (2014). Relación de las empresas con violación al Reglamento 522-06, sobre Seguridad y Salud en el Trabajo. Santo Domingo: Ministerio de Trabajo.

República Dominicana. (2014). Estadísticas. Recuperado de http://www.sisalril.gov.do/

Vílchez, L. (2014, 7 de junio). Nuevas formas de hostigar a las Pymes. Diario Libre, p. 12. 


\section{Yuri Alberto Durán Rodríguez}

Es ingeniero industrial egresado de INTEC, en 1991; maestría en Alta Gerencia (INTEC, 1999); máster en Prevención de Riesgos Laborales por la Universidad A Coruña, 2003. Se ha desempeñado como:

- Director Ejecutivo de la Red Nacional de Apoyo Empresarial a la Protección Ambiental (RENAEPA octubre 2008-julio, 2010).

- Catedrático de Maestría en Prevención de Riesgos Laborales de INTEC (actual).

Publicaciones recientes:

- Durán Rodríguez, Y. A. (2008). Contribución a la formulación de una política nacional de prevención”. Ciencia y Sociedad, 28(2), 2008, 166-192.

- Durán Rodríguez, Y. A. (2009). Capacidades del sector privado para la implementación del Reglamento de seguridad y salud en el trabajo de República Dominicana, Ciencia y Sociedad, 34(3), 388-404.

Correo electrónico: yuriduran@passa.com.do, yuri.duran@intec.edu.do

Recibido: 10-12-2015

Aprobado: 12-06-2016 